\title{
Better Distance Running Through Strength Training Minimizing Muscle Truama
}

\author{
Dr. Okundare ${ }^{1}$, Dr. Osigwe ${ }^{2}$, Dr. Okueso ${ }^{3}$, \\ ${ }^{1}$ Ayobami Alade, Olabisi Onabanjo University, Ago-Iwoye, Ogun State. Nigeria. \\ ${ }^{2}$ Cyril Abel, University Of Agriculture Abeokuta, Abeokuta, Ogun State, Nigeria. \\ ${ }^{3}$ Samuel Adesina Olabisi Onabanjo University, Ago-Iwoye, Ogun State, Nigeria.
}

\begin{abstract}
Strength training is an essential element of fitness for virtually every sports man and woman. Long gone are the days when coaches believed strength training (resistance exercises) only added unnecessary bulk to the athlete, hindering their ability to execute skill, not knowing that strength training prevent injury. Injury rates among runners are extremely high. In fact, at beginner's level distance runners experience more injuries than athletes in any other sport, including football and gymnastics. This is because running involves an incredible amount of contact with soft and hard surfaces rather than other athletes. Every running stride places about three times the weight of your body on your foot, ankle, knee and hip joints. These landing forces stress your body structures. The repetitive pounding encountered mile after mile produces a degree of microtrauma to the shock-absorbing tissues. Under ideal conditions, these tissues recover completely within a 24-hour period. However, there are numerous factors that may interfere with normal recovery processes, eventually resulting in weakened and injury-prone tissues. These factors include longer running sessions, faster running paces, shorter recovery periods between workouts, more downhill running, more hard-surface running, more racing, more general fatigue, and undesirable changes in eating or sleeping patterns. This paper wisely looked into the most effective means for minimizing tissue trauma which is to develop stronger muscles, tendons, fascia, ligaments and bones through strength exercise training.
\end{abstract}

Keywords: WordCount: 227

\section{Introduction}

Running is a means of terrestrial locomotion allowing humans to move rapidly on foot. It is simply defined in athletics terms as a gait in which at regular points during the running cycle both feet are off the ground. This is in contrast to walking, where one foot is always in contact with the ground, the legs are kept mostly straight and the center of gravity vaults over the legs in an inverted pendulum fashion (Biewener. 2003). A characteristic feature of a running body from the viewpoint of spring-mass mechanic is that changes in kinetic and potential energy within a stride occur simultaneously, with energy storage accomplished by springy tendons and passive muscle elasticity (http://jap.physiology.org/cgi/content/abstract/19/2/249). The term running can refer to any of a variety of speeds ranging from jogging to sprinting, sprinting and distance running.

Distance running is a great sport that is enjoyed at a variety of levels by millions of competitive sports, games and recreational athletes. Whether you prefer to jog a couple of miles through the neighborhood, or you are training to complete a marathon, distance running is a highly effective and efficient means of aerobic conditioning and considerably less beneficial for your musculoskeletal system. Injury rates among runners are extremely high. In fact, at beginner's level distance runners experience more injuries than athletes in any other sport, including football and gymnastics (Lombardi and Patteson, 2009).

People do ask the question: Why is a non-contact sport like running such a high-risk activity? Actually, running involves an incredible amount of contact with surfaces rather than other athletes. Every running stride places about three times the weight of your body on your foot, ankle, knee and hip joints. These landing forces may also stress your lower back/body structures (Johnston, Quinn, Kertzer, and Vroman. 1997).

The repetitive pounding encountered mile after mile produces a degree of microtrauma (shock) to the shock-absorbing tissues. Under ideal conditions, these tissues recover completely within a 24-hour period (Powers, Scott and Howley, Edward, 2003). Because of its high-impact nature, many injuries are associated with running. They include "runner's knee" (pain in the knee), shin splints, pulled muscles (especially the hamstring), twisted ankles, iliotibial band syndrome, plantar fasciitis, Achilles tendinitis, and stress fractures. Repetitive stress on the same tissues without enough time for recovery or running with improper form can lead to many of the above (Herbert \& Gabriel. 2002). There are numerous factors that may interfere with normal recovery processes, eventually resulting in weakened and injury-prone tissues. These factors include longer running sessions, faster running paces, shorter recovery periods between workouts, more downhill running, more hard-surface running, more racing, more general fatigue, and undesirable changes in eating or sleeping patterns (Powers, Scott, Howley, Edward, 2003). 
You may wisely take steps to reduce the amount of tissue trauma and decrease your risk of runningrelated injuries. Such precautions include making very gradual increases in training distances and speeds, taking sufficient recovery periods (particularly between hard strength training sessions), selecting user-friendly running courses (soft surfaces and level terrain), competing in fewer races, avoiding over-fatigue and paying careful attention to proper nutrition and sleep (Campos GE, Luecke TJ, Wendeln HK, 2002)\& (Donatelle, Rebecca J, 2005). However, one of the most effective means for minimizing tissue trauma is to develop stronger muscles, tendons, fascia, ligaments and bones through strength training (Delavier \&, Frederic 2001). The concept below is the primary reason why every runner should perform regular strength training exercise.

\section{Concept of Strength Training}

Strength training is a vital part of a balanced exercise routine that includes aerobic activity and flexibility exercises. Regular aerobic exercise, such as running or using a stationary bike, makes your muscles use oxygen more efficiently and strengthens your heart and lungs (Cooper \& Kenneth C, 2005). It is good to know that weight training is strength training. When you strength train with weights, you're using your muscles to work against the extra $\mathrm{Kg}$ (this concept is called resistance). This strengthens and increases the amount of muscle mass in your body by making your muscles work harder than they're used to (Hoff J, Gran A, Helgerud J. 2002). Most people who work out with weights typically use two different kinds: free weights (including barbells, dumbbells, and hand weights) and weight machines. Free weights usually work a group of muscles at the same time; The weight machines typically are designed to help you isolate and work on a specific muscle. Most gyms or weight rooms set up their machines in a circuit, or group, of exercises that you perform to strengthen different groups of muscles (Kraemer, William J.; Zatsiorsky, Vladimir M. 2006).

People can also use resistance bands and even their own body weight (as in pushups, sit-ups, or body weight squats) for strength training. Many people tend to lump all types of weightlifting together, but there's a big difference between strength training, powerlifting, and competitive bodybuilding! When lifting weights either free weights or on a machine make sure that there's always someone nearby to supervise, or spot, you. This person, called a spotter, encourages you and also can act as your coach, telling you if you're not doing a particular exercise correctly (Andersen, R.E.; Jakicic, J.M. 2003). Having a spotter nearby is particularly important when using free weights. Even someone in great shape sometimes just can't make that last rep. It's no big deal if you're doing bicep curls; all you'll have to do is drop the weight onto the floor. But if you're in the middle of a bench press - a chest exercise where you're lying on a bench and pushing a loaded barbell away from your chest - it's easy to become trapped under a heavy weight. A spotter can keep you from dropping the barbell onto your chest (Joseph.A and Congeni,M D 2009).

The benefits of strength training to athletic performance are enormous and many. Not only is an integral conditioning component for power athletes such as football and rugby players, performance in the pure endurance events can be improved with a well-structured strength routine (Hoff J \& Gran A, Helgerud J 2002). However, aside from perhaps bodybuilders, sport-specific resistance training requires a more refined approach than simply lifting heavy weights to complete exhaustion. A physiological analysis of any game or event will confirm that most athletes require explosive power, muscular endurance, maximal strength or some combination of all three in order to excel. Rarely is pure muscle bulk the primary concern and when it is, other elements of strength are equally as important (Ormsbee, et-al 2007).

\section{Strength Training Methods}

Strength training uses resistance methods like free weights, weight machines, resistance bands, or a person's own weight to build muscles and strength. Olympic lifting, or powerlifting, which people often think of when they think of weightlifting, concentrates on how much weight a person can lift at one time. Competitive bodybuilding involves evaluating muscle definition and symmetry, as well as size (Kraemer, William J.; Zatsiorsky, Vladimir M. 2006). Powerlifting, competitive weightlifting, and bodybuilding are not recommended for teens who are still maturing. That's because these types of activity can cause serious injuries to growing bones, muscles, and joints (Dowshen, S; Homeier B, 2008).

\section{Here are some basic rules to follow in strength training:}

i. Start with body weight exercises for a few weeks (such as sit-ups, pushups, and pull-ups) before using weights.

ii. Work out with weights about three times a week. Avoid weight training on back-to-back days.

iii. Warm up for 5-10 minutes before each session.

iv. Spend no more than 40 minutes in the weight room to avoid fatigue or boredom.

v. Work more reps; avoid maximum lifts.

vi. Ensure you're using proper technique through supervision. Improper technique may result in injuries, particularly in the shoulder and back.

vii. Cool down for 5-10 minutes after each session, stretching the muscles you worked out. 


\section{How Does Resistance Exercise Work?}

Resistance training works by causing microscopic damage or tears to the muscle cells, which in turn are quickly repaired by the body to help the muscles regenerate and grow stronger. The breakdown of the muscle fiber is called "catabolism," and the repair and re-growth of the muscle tissue is called "anabolism."

You are probably familiar with the term anabolic when used with steroids. Anabolic means to grow, and that's exactly what happens after you break down the muscle fibers with resistance exercise. In fact, many biological processes of growth in the body require some breakdown, or catabolism, prior to re-growth. For instance, bones must be broken down first before calcium and other growth factors repair the bone and make it stronger. With muscles, testosterone, insulin-like growth factor, growth hormone, protein, and other nutrients rush to the muscle after a resistance-exercise session to help repair the muscles to make them stronger. Importantly, your muscles heal and grow when you aren't working out, and so that's why it's necessary to leave time between workouts for recovery (Joseph.A and Congeni,M D 2009).

\section{The University of Agriculture Abeokuta, Abeokuta Strength Training Programme}

For 12 weeks, 15 distance runners (10 males, 5 females) from The University of Abeokuta participated in a basic and brief strength training programme. Every Mondays, Wednesdays, and Fridays, they performed 30 minutes of strength exercise (12 stations Nautilus machines) that addressed all of their major muscle groups. Each of these months improvements in their timings were observed and no injury was recorded.

\section{Strength Training Benefits}

These athletes realized that this strength training programme provides many benefits for them. These includes:

a. Greater muscle strength

b. Greater muscle endurance

c. Greater joint flexibility

d. Better body composition

e. Reduced injury risk

f. Improved self-confidence

g. Improved running economy

While the first six strength training benefits should be self-explanatory, you may be intrigued by improved running economy. In a 1995 study at the University of New Hampshire, the women cross-country runners who did strength training experienced a significant improvement in their running economy. They required 4 percent less oxygen at sub-maximum running speeds (7:30, 7:00, and 6:30 minute mile paces), meaning that they could run more efficiently and race faster than before (Johnston RE, TJ Quinn, Kertzer $R$ and Vroman NB. 1997).

\section{Misconceptions and Runner Concerns}

Consider these four concerns that keep many runners from strength training which were revealed through findings from runners:

a. Increased Bodyweight

b. Decreased movement speed

c. Less fluid running form

d. Fatigued muscles

Let us take a closer look at each of these issues.

\section{a. Increased Bodyweight}

Very few people who perform strength exercise have the genetic potential to develop large muscles. This is especially true for distance runners, who typically have ectomorphic (thin) physiques. Strength training increases their muscle strength and endurance, but rarely results in significant weight gain ( Ada, Dorsch and Canning, 2006).

\section{b. Decreased Movement Speed}

With respect to running speed, our studies and many others have Sshown that greater strength results in faster movement speeds. We need only look at sprinters and middle distance runners to realize that strength training has a positive impact on running speed, as essentially all of these athletes perform regular strength exercise. (Kraemer, William, Zatsiorsky and Vladimir. 2006)

\section{c. Less Fluid Running Form}

Running involves coordinated actions of the legs and the arms, and one cannot function without the other. Your right arm moves in mirror image with your left leg, and your left arm counterbalances your right leg 
in perfect opposition. That is why it is almost impossible to run fast and move your arms slow or to move your arms fast and run slow. By strengthening the upper body muscles, you more effectively share the running effort between your arms and legs, resulting in more fluid running form (Rønnestad, Egeland, Kvamme, Refsnes, Kadi, Raastad, 2007).

\title{
d. $\quad$ Fatigued Muscles
}

It is true that a strenuous strength training session can cause a considerable amount of muscle fatigue that could adversely affect the quality and quantity of your runs. That is why we recommend brief strength workouts that do not leave you feeling enervated or exhausted. Remember that you are strength training to enhance your running performance, not to become a competitive weightlifter. Our program of strength training requires just one set of exercise for each major muscle group, which does not take much time and does not produce much lasting fatigue. You may also choose to strength train only one or two days per week, which should make muscle fatigue even less likely.

\section{Runners' Strength Training Program}

The strength training protocol followed by the University of agriculture Abeokuta athletes, is a comprehensive conditioning program that addresses all of the major muscle groups in the body. We do not attempt to imitate specific running movements or emphasize specific running muscles, because this typically results in an overtrained, imbalanced, and injury-prone musculoskeletal system. For example, the calf (gastrocnemius and soleous) muscles are used extensively in running. Due to their involvement in every running stride, many people think that runners should strengthen their calf muscles. Indeed they should, but it is even more important to strengthen their weaker counterpart, the shin (anterior tibial) muscles. If you strengthen only the larger and stronger calf muscles they will eventually overpower the smaller and weaker shin muscles, which may lead to shin splints, stress fractures, achilles tendon problems, and other lower leg difficulties. With this in mind, our runners always conclude their strength workouts with a set of weighted toe raises to strengthen the shin muscles and maintain balance within the lower leg musculature.

Some people believe that runners should complete numerous sets and many repetitions with light resistance to enhance their endurance capacity. However, this was not our purpose in performing strength training. Remember that running is best for improving cardiovascular endurance, and that strength training is best for increasing musculoskeletal strength. Muscle strength is best developed by training with moderate weight-loads (about 75 percent of maximum) for 8 to 12 repetitions per set (Johnston RE, TJ Quinn, Kertzer R and Vroman NB. 1997. However, distance runners typically possess a higher percentage of slow-twitch muscle fibers, and therefore attain better results by training with about 12 to 16 repetitions per set. You should add 1 to $5 \mathrm{Kg}$ more resistance whenever you complete 16 repetitions in good form. One set of each exercise is sufficient for strength development (Herbert, R.D. \& Gabriel, M. 2002). There is no reason to train with fast movement speeds, because training fast will not make you faster and training slow will not make you slower. Exercising with controlled movement speeds maximizes muscle tension and minimizes momentum for a better training effect. We used six-second repetitions, taking two seconds for each lifting movement and four seconds for each lowering movement.

Research supports three non-consecutive strength training sessions per week for best results, but fewer workouts can produce significant strength gains. Our recent studies have shown two weekly workouts to be 70 to 85 percent as effective and one weekly workout to be 60 to 75 percent as effective as three-day-per-week strength training (Johnston RE, TJ Quinn, Kertzer R and Vroman NB. 1997).

\author{
Summary of Strength Training Guidelines \\ i. Exercise all of the major muscle groups \\ ii. Perform 12 to 16 repetitions per set \\ iii. Add one to five Kgs whenever 16 repetitions can be completed \\ iv. Perform one set of each exercise \\ v. Use controlled movement speeds (six seconds per rep) \\ vi. Train one, two or three non-consecutive days per week
}

\section{Recommended Strength Exercises For Runners}

You may develop muscle strength with a variety of exercises using free-weights or machines. The following section presents recommended strength exercises for the major muscle groups (Fleck SJ and Kraemer WJ. 2004). 


\section{Leg Muscles}
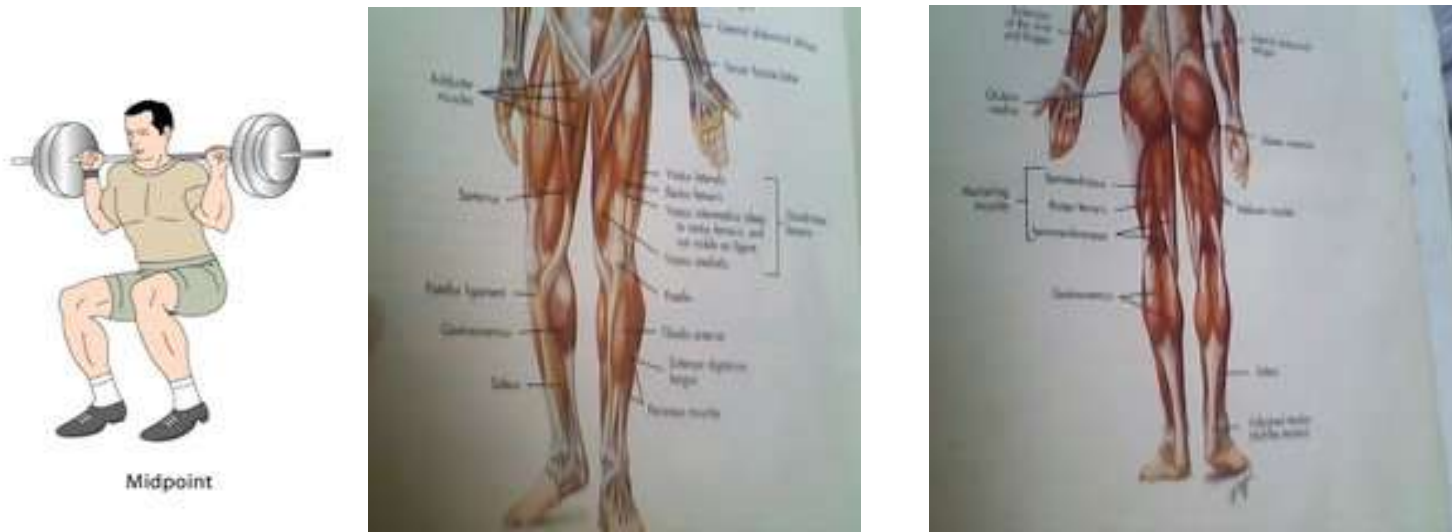

Although barbell squats are the traditional leg exercise, most runners may do better to avoid placing a heavy barbell across their shoulders. Dumbbell squats are an acceptable alternative, but it may be difficult to hold enough weight to appropriately stress the large leg muscles.

The recommendation is leg presses on a well-designed machine that offers a full movement range and good back support. It may be advisable to precede leg presses with leg extensions that target the quadriceps and leg curls that target the hamstrings. One set of each exercise is sufficient, but you may perform an additional set if you desire.

\section{Upper Body Muscles}
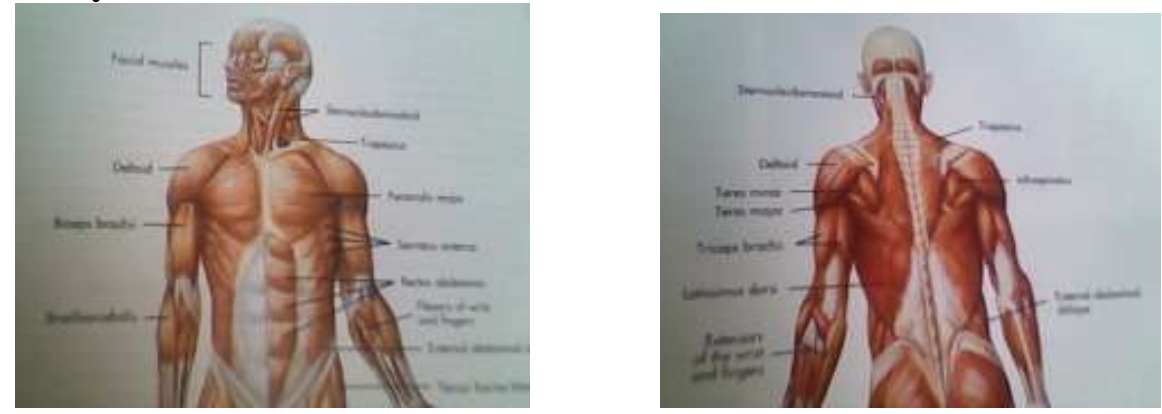

The typical exercises for the upper body are bench presses for the chest muscles, bent rows for the midupper back muscles, and overhead presses for the shoulder muscles. These are acceptable exercises, but are much safer when performed with dumbbells rather than barbells. For example, because there is no back support in a barbell bent row, the stress to the low-back area is 10 times the weight of the barbell. By using one dumbbell, and placing your other hand on a bench for back support, this exercise can be performed more safely and effectively.If you have access to machines, chest crosses for the chest muscles, pullovers for the mid and upper back muscles, and lateral raises for the shoulder muscles is recommended. These machines require rotary movements that better isolate the target muscle groups. If you prefer linear movements that involve more muscle groups, well-designed chest press, seated row, and shoulder press machines provide combined training for the upper body and arm muscles.

\section{Arm Muscles}
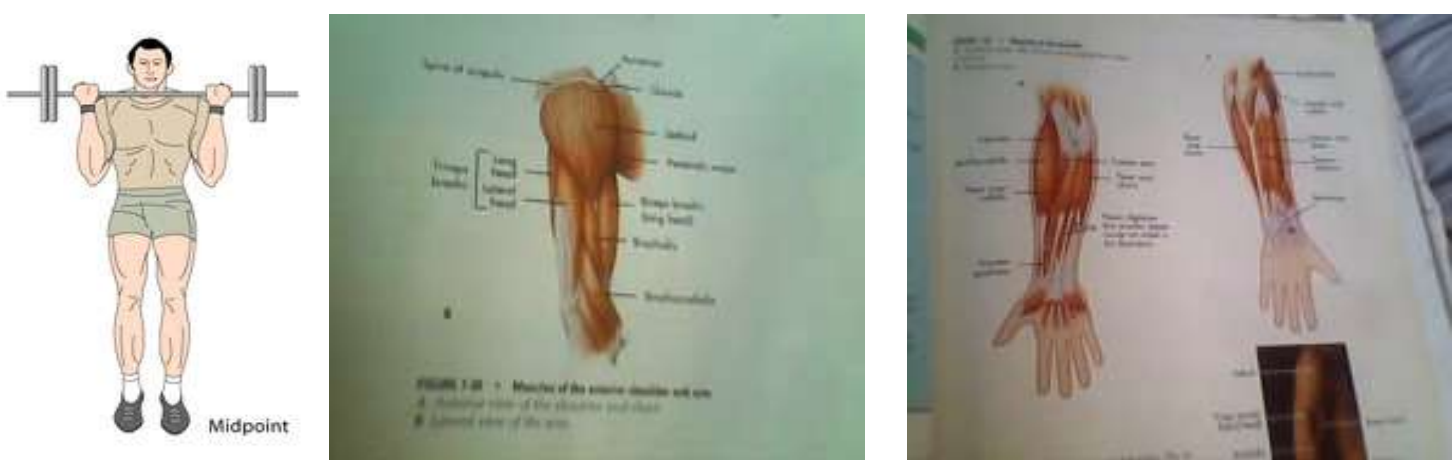
The basic exercise for the biceps muscles is the arm curl, performed with barbells, dumbbells, or machines. Training the triceps involves some form of arm extension, either with free-weights or machines.

A good means for working the biceps and upper back muscles together is chin-ups with bodyweight or on a weight-assisted chin/dip machine. A good means for working the triceps and chest muscles together is bar dips with bodyweight or on a weight-assisted chin/dip machine.

\section{Midsection Muscles}
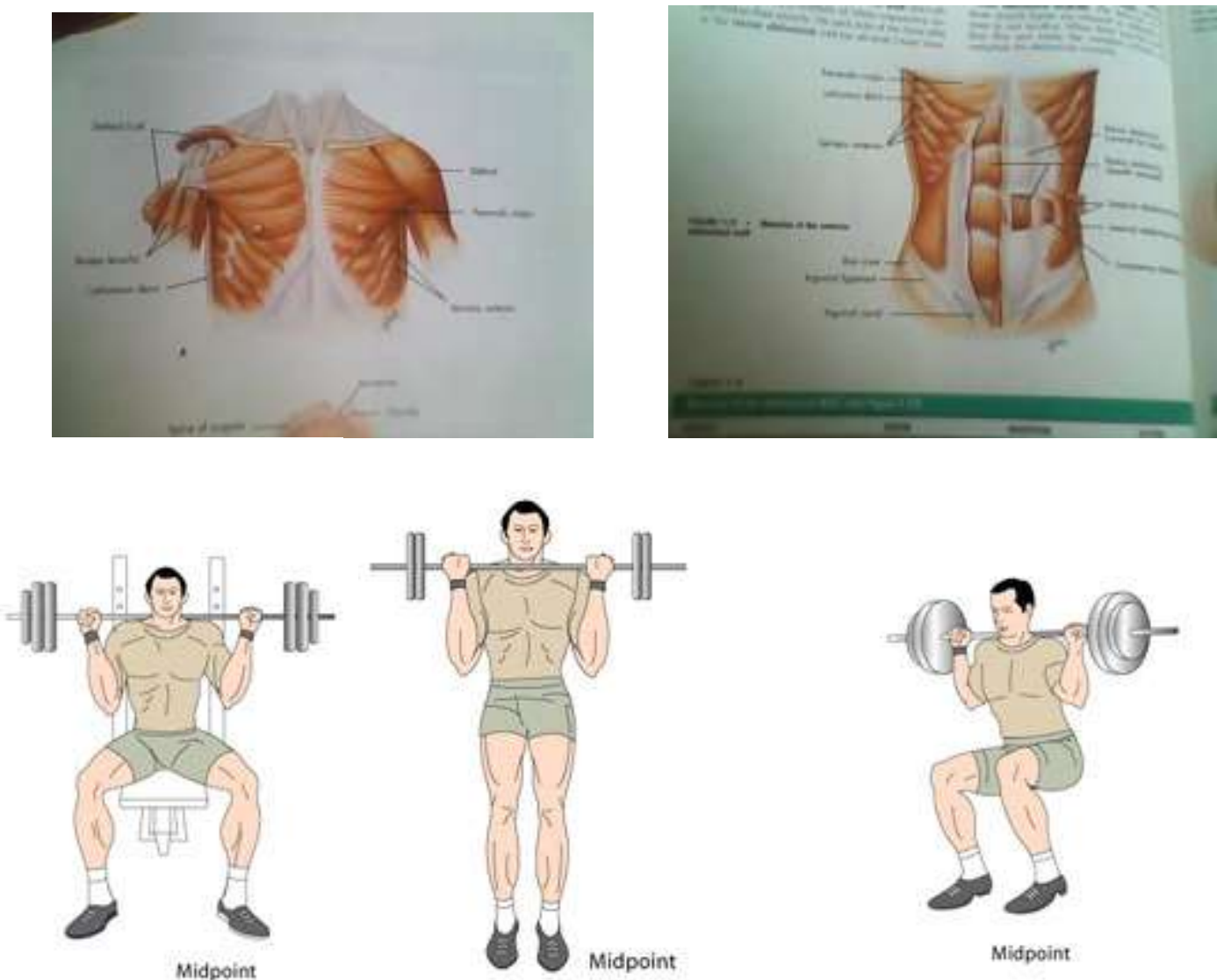

Machines provide the best means for safely and progressively conditioning the muscles of the midsection. The abdominal machine and low-back machine are key exercises for developing a strong and injury resistant midsection. We also recommend the rotary torso machine for strengthening the oblique muscles surrounding the midsection.

If appropriate machines are not available, the basic trunk curl may be the best alternative for abdominal conditioning. The recommended counterpart for the low-back muscles is a front-lying (face down) back extension. Although both of these exercises are performed with bodyweight resistance they are reasonably effective for strengthening the midsection muscles.

\section{Neck Muscles}

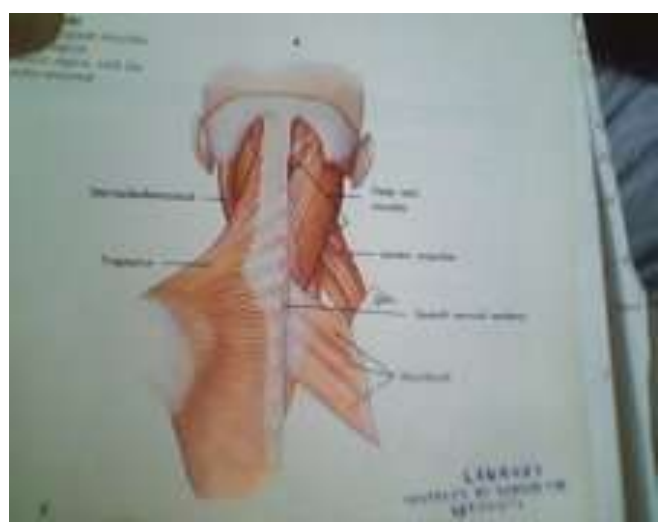


The neck muscles maintain head position throughout each run. As the head weighs up to $15 \mathrm{Kg}$, this is an important function. In fact, the first place where many runners fatigue and tighten up is the neck/shoulder area. The 4-way neck machine to strengthen these muscles was recommended. If you do not have access to this machine, perhaps the best approach is manual resistance. That is, place your hands in front of your forehead to resist slow neck flexion movements, and place your hands behind your head to resist slow neck extension movements.

\section{Muscle of the Head}
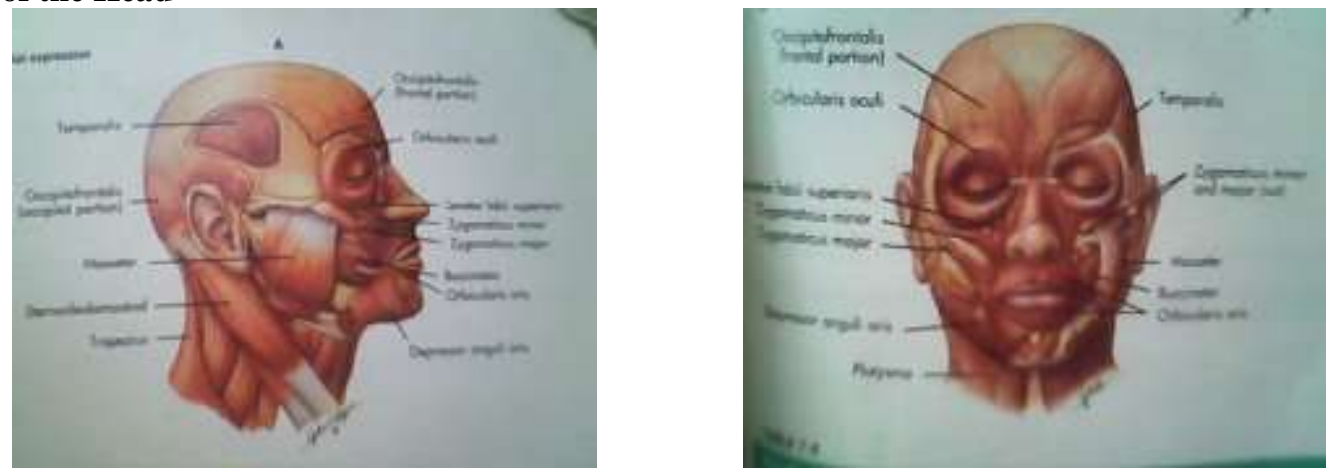

Table 1 presents the recommended strength training exercises for an overall conditioning program that should be beneficial for runners (Fleck SJ and Kraemer WJ. 2004).

Table 1. Recommended Strength Training Program: Basic Exercises

\begin{tabular}{|l|l|l|}
\hline Major Muscle Groups & Machine Exercises & Free-Weight Exercises \\
\hline Quadriceps & Leg Extension Machine & Dumbbell Half-Squat \\
\hline Hamstrings & Leg Curl Machine & Dumbbell Half-Squat \\
\hline Chest & Chest Cross Machine & Dumbbell Bench Press \\
\hline Upper Back & Pullover Machine & Dumbbell Bent Row \\
\hline Shoulders & Lateral Raise Machine & Dumbbell Overhead Press \\
\hline Biceps & Biceps Machine & Dumbbell Biceps Curl \\
\hline Triceps Extension & Triceps Machine & Dumbbell Triceps \\
\hline Low Back & Low Back Machine & Back Extension (Bodyweight) \\
\hline Abdominals & Abdominal Machine & Trunk Curls (Bodyweight) \\
\hline
\end{tabular}

Once you have mastered the basic exercise program, you may want to add some of the exercises presented in Table 2.

Table 2. Recommended Strength Training Program: Additional Exercises

\begin{tabular}{|l|l|l|}
\hline Muscle Groups & Machine Exercises & Free Weight Exercises \\
\hline Quadriceps\& Hamstrings & Leg Press Machine & Dumbbell Lunge \\
\hline Chest \& Triceps & Weight-Assisted Chin/Dip Machine & Bar Dip \\
\hline Upper Back \& Biceps & Weight-Assisted Chin/Dip Machine & Chin Up \\
\hline Internal\&External Obliques & Rotary Torso Machine & Trunk Curls w/Twists \\
\hline Neck Flexors\& Extensors & 4-Way Neck Machine & Manual Resistance Neck \\
\hline & & Flexion \& Extension \\
\hline Calves & Calf Machine & Dumbbell Heel Raises \\
\hline Shins & & Weight Plate Toe Raises \\
\hline
\end{tabular}

\section{Conclusion}

The main objective of a strength training program for runners is to decrease injury risk and increase performance potential. For best results the program should be high in exercise intensity and low in training time. One good set of 12-16 repetitions for each major muscle group is recommended for a safe, effective and efficient exercise experience. One or two training sessions per week are sufficient, although three weekly workouts produces greater strength gains. Each workout should take no more than 20-30 minutes depending upon the number of exercises performed. The key to productive strength training is proper exercise technique, which includes full movement range and controlled movement speeds. When you make every repetition count, a basic and brief training program should increase your strength significantly (40-60 percent) over a two month training period. 


\section{References}

[1] Ada L, Dorsch S, Canning C G ( 2006). "Strengthening interventions increase strength and improve activity after stroke: a systematic review " Australian Journal of Physiotherapy. ;52(4):241-248.

[2] Andersen, R.E.; Jakicic, J.M. (2003). "Physical activity and weight management: Building the case for exercise". The Physicial and Sportsmedicine 31 (9).

[3] Biewener, A. A. (2003). Animal Locomotion. Oxford University Press, USA. ISBN 978-0198500223, Campos GE, Luecke TJ, Wendeln HK, et al. (2002). "Muscular adaptations in response to three different resistance-training regimens: specificity of repetition maximum training zones". Eur. J. Appl. Physiol. 88 (1-2): 50-60.

[4] Delavier, Frederic (2001). Strength Training Anatomy. Human Kinetics Publishers. ISBN 0-7360-4185-0.

[5] Cooper, Kenneth C. (2005). The New Aerobics. Eldora, Iowa: Prairie Wind.

[6] Donatelle, Rebecca J. (2005) Health: The Basics. 6th ed. San Francisco: Pearson Education, In Delavier, Frederic (2001). Strength Training Anatomy.Human Kinetics Publishers. ISBN 0-7360-4185-0.

[7] De Mello Meirelles, C.; Gomes, P.S.C. (2004). "Acute effects of resistance exercise on energy expenditure: revisiting the impact of the training variables". Rev Bras Med Esporte 10: 131-8.

[8] Dowshen, S; Homeier B (2008). "Strength Training and Your Child". kidshealth.org. http://kidshealth.org/parent/nutrition_fit/fitness/strength-training.html.

[9] Fleck SJ and Kraemer WJ. (2004) Designing Resistance Training Programs: 3rd Edition. Champaign, IL: Human Kinetics

[10] Herbert, R.D. \& Gabriel, M. (2002). Effects of stretching before and after exercising on muscle soreness and risk of injury: systematic review. British Medical Journal, 325 p. 468).

[11] Hoff J, Gran A, Helgerud J. (2002) Maximal strength training improves aerobic endurance performance. Scand J Med Sci Sports. Oct;12(5):288-95 http://jap.physiology.org/cgi/content/abstract/19/2/249

[12] Johnston RE, TJ Quinn, Kertzer R and Vroman NB. (1997) Strength training in female distance runners: impact on running economy. J. Strength Cond. Res. 11: 224-229

[13] Joseph A and Cogeni MD, (2009). 'Strength Training' http//kidhealth.org/tean/kh misc/send mail-Kraemer, William J.; Zatsiorsky, Vladimir M. (2006). Science and Practice of Strength Training, Second Edition. Champaign, Ill: Human Kinetics Publishers.

[14] Ormsbee, Michael J.; Ormsbee, John P. Thyfault, Emily A. Johnson, Raymond M. Kraus, Myung Dong Choi, Robert C. Hickner (May 2007). "Fat metabolism and acute resistance exercise in trained men". Journal of Applied Physiology 102 (5): $1767-1772$.

[15] Rønnestad BR, Egeland W, Kvamme NH, Refsnes PE, Kadi F, Raastad T (2007). "Dissimilar effects of one-and three-set strength training on strength and muscle mass gains in upper and lower body in untrained subjects". J Strength Cond Res 21 (1): 157-63.

[16] Rippetoe, Mark and Kilgore, Lon (2007) "Starting Strength (2nd Edition)". The Aasgaard Company.

[17] Schoenfeld, Brad (2002). Sculpting Her Body Perfect. Human Kinetics Publishers.

[18] Lombardi, V. Patteson (2009). Beginning Weight Training. Wm. C. Brown Publishers. ISBN 0-697-10696-9 